\title{
Noncompact Perturbation of Sweeping Process with Delay in Banach Spaces
}

\author{
F. Aladsani and A. G. Ibrahim \\ Department of Mathematics, Faculty of Science, King Faisal University, AL-Ahsa 31982, Saudi Arabia \\ Correspondence should be addressed to A. G. Ibrahim; agamal2000@yahoo.com
}

Received 20 June 2013; Accepted 23 July 2013

Academic Editor: Jean Michel Rakotoson

Copyright (C) 2013 F. Aladsani and A. G. Ibrahim. This is an open access article distributed under the Creative Commons Attribution License, which permits unrestricted use, distribution, and reproduction in any medium, provided the original work is properly cited.

\begin{abstract}
We have proven an existence theorem concerning the existence of solutions for a functional evolution inclusion governed by sweeping process with closed convex sets depending on time and state and with a noncompact nonconvex perturbation in Banach spaces. This work extends some recent existence theorems concerning sweeping processes from Hilbert spaces setting to Banach spaces setting. Moreover, it improves some recent existence results for sweeping processes in Banach spaces.
\end{abstract}

\section{Introduction}

Differential inclusions represent an important generalization of differential equations. Moreover, they have several applications in different branches of mathematical sciences such as Control Theory, Viscosity, Optimization, and Mechanical problems (see [1-4]). In his leading paper, Moreau [5] proposed and studied the following differential inclusion governed by sweeping process of first order:

$$
\begin{gathered}
-u^{\prime}(t) \in N_{G(t)}(u(t)) \quad \text { a.e. on } I=[0, T], \\
u(0)=u_{0},
\end{gathered}
$$

where $G$ is a set-valued function from the interval $I=[0, T]$ to the family of nonempty closed convex subsets of a Hilbert space $H$ and $N_{G(t)}(u(t))$ is the normal cone of the subset $G(t)$ at the point $u(t)$. Problem (1) corresponds to several important mechanical problems. For more details concerning the applications of (1), we refer to [5].

Since then, important improvements have been developed by weakening assumptions in order to obtain the most general result of existence for sweeping processes.

Let $X$ be a Banach space, $r$ a positive real number, $C_{r}=C_{X}([-r, 0])$ the Banach space of continuous functions from $[-r, 0]$ to $X$ endowed with the uniform norm $\|u\|_{\infty}=$ $\operatorname{Sup}\{\|u(t)\|: t \in[-r, 0]\}, C$ a multifunction from $I \times X$ to the family of nonempty closed convex subsets of $X$, and $F$ a multifunction defined on $[0, T] \times C_{X}([-r, 0]) \times C_{X}([-r, 0])$ and with nonconvex noncompact values in the dual space, $X^{*}$, of $X$. The aim of this paper is to find the sufficient conditions that guarantee the existence of two continuous functions $u:[-r, T] \rightarrow X^{*}$ and $w:[-r, T] \rightarrow X$ such that the following perturbed sweeping process is satisfied:

$$
\begin{gathered}
u(t)=J(\varphi(t)), \quad w(t)=\psi(t), \quad \text { for } t \in[-r, 0], \\
u(t)=J(\varphi(0))+\int_{0}^{t} u^{\prime}(s) d s, \quad \text { for } t \in I, \\
J^{*}(u(t)) \in C(t, w(t)), \quad \text { for } t \in I, \\
w(t)=\psi(0)+\int_{0}^{t} J^{*} u(s) d s, \quad \text { for } t \in I, \\
u^{\prime}(t) \in-N_{C(t, w(t))}\left(J^{*} u(t)\right)+F\left(t, \tau(t) w, \tau(t) J^{*} u\right), \\
\text { a.e. for } t \in I,
\end{gathered}
$$

where $\varphi, \psi \in C_{X}([-r, 0])$ are given with $\varphi(0) \in C(0, \psi(0))$, $J$ is the duality mapping in $X$, and for each $t \in[0, T], \tau(t)$ : $C_{X}([-r, t]) \rightarrow C_{X}([-r, 0]),(\tau(t) g)(s)=g(s+t)$, for all $s \in$ $[-r, 0]$.

In order to explain the mathematical motivation for this work, we mention some recent results in this domain. In 
a very recent paper, Aitalioubrahim [6] considered (2) when $X$ is a Hilbert spaces $C$ is a multifunction from $I$ to the family of nonempty closed nonconvex subsets of $X$, and $F$ is a multifunction defined on $[0, T] \times C_{X}([-r, 0])$ and with nonconvex noncompact values in $X$. Castaing et al. [7] considered a second order sweeping process without delay in a separable Hilbert space $H$ in the case when $C$ is a Lipschitz multifunction defined on $I \times H$, taking a closed $\rho$-proxy regular $(\rho>0)$ values in $H$, and $F$ is a convex weakly compact valued scalary uppersemicontinuous defined on $\mathrm{I} \times \mathrm{H} \times \mathrm{H}$ and satisfying the growth condition

$$
F(t, x, y) \subseteq(1+\|x\|+\|y\|) B_{H},
$$

for all $(t, x, y) \in[0, T] \times H \times H$. For other results concerning (2) with or without perturbation in Hilbert spaces, we refer to [8-13]. Bounkhel and Al-Yusof [14] initiated the extension of (1) from the Hilbert setting to the Banach spaces setting. In fact, Bounkhel [8] considered (2) when $C$ is a multifunction from $I$ to the family of nonempty closed convex subsets of $X$ and satisfyes a condition similar to Condition $\left(C_{1}\right)$ in the statement of our result (Theorem 18) and $F$ is an uppersemicontinuous multifunction defined on $[0, T] \times X$ and with convex compact values in $X^{*}$ such that $F(t, x) \subseteq Z$, for all $(t, x) \in[0, T] \times X$, for some convex compact set $Z \subset X^{*}$. More recently, Ibrahim and Aladsani [15] considered a second order sweeping process without delay in a separable $p$-uniformly convex and $q$-uniformly smooth Banach space $X$, and the perturbation $F$ is an upper semicontinuous defined from $[0, T] \times X \times X$ to the family of nonempty convex weakly compact sets of the topological dual space $X^{*}$ of $X$ such that

$$
\begin{gathered}
F(t, x, y) \subseteq(1+\|x\|+\|y\|) B_{X}, \\
\forall(t, x, y) \in[0, T[\times X \times X .
\end{gathered}
$$

In this paper, our main purpose is to prove the existence of solutions of (2) in the case when the perturbation $F$ is nonconvex noncompact values. We will adapt the discretization technique used in [6].

The paper is organized as follows. Section 2 is devoted to some definitions and notations needed later. In Section 3, we prove in Theorem 18 the main result.

We note that if $X$ is a Hilbert space, then $J$ is equal to the identity operator on $X$, and $X$ is 2-uniformly convex and 2uniformly smooth Banach space. Therefore, our work extends many problems from Hilbert spaces setting to Banach spaces setting. Moreover, our work improves many results in the literature concerning the existence of solutions for some evolution inclusions governed by sweeping process in Banach spaces, for example, $[14,15]$. In addition our technique allows us to discuss some sweeping process problems with noncompact perturbation in Banach spaces.

\section{Preliminaries and Notations}

Let $I=[0, T], T>0$, and let $X$ be a Banach space with topological dual space $X^{*}$. Let $B=\{x \in X:\|x\| \leq 1\}$ and $B_{*}=\left\{z \in X^{*}:\|z\| \leq 1\right\}$.
Definition 1 (see [1, Definition 2.2.1]). A Banach space $X$ is said to be uniformly convex if for any $\varepsilon, 0<\varepsilon \leq 2$, the inequalities $\|x\| \leq 1,\|y\| \leq 1$, and $\|x-y\| \geq \varepsilon$ imply that there exists a $\delta=\delta(\varepsilon)$ such that $\|(1 / 2)(x+y)\| \leq 1-\delta$.

Lemma 2 (see [16, Theorems 2.2.4 and 2.2.8]).

(1) Every Hilbert space is uniformly convex.

(2) Every uniformly convex space is strictly convex.

(3) Every uniformly convex space is reflexive.

Definition 3 (see $[1,17,18]$ ). Let $p>1$ be a real number. A Banach space $X$ is said to be $p$-uniformly convex if there exists a constant $\lambda>0$ such that

$$
\delta_{X}(\varepsilon) \geq \lambda \varepsilon^{p}, \quad \forall \varepsilon \in(0,2] .
$$

Definition 4 (see [1, Definition 2.4.1]). Let $X^{*}$ be the topological dual of a Banach space $X$; then the multivalued mapping

$$
\begin{gathered}
J: X \longrightarrow 2^{X^{*}}, \\
J(x)=\left\{y \in X^{*}:\langle x, y\rangle=\|x\|^{2}=\|y\|^{2}\right\}
\end{gathered}
$$

is said to be the duality mapping (or duality mapping) in $X$, and the multivalued mapping

$$
\begin{gathered}
J^{*}: X^{*} \longrightarrow 2^{X}, \\
J^{*}(y)=\left\{x \in X:\langle y, x\rangle=\|x\|^{2}=\|y\|^{2}\right\}
\end{gathered}
$$

is called the normalized duality mapping (or duality mapping) in $X^{*}$.

In the following lemma, we recall some properties of the duality mapping.

Lemma 5 (see [1, 19] Propositions 2.4.5, 2.4.12, and 2.4.15).

(1) If $X$ is a Hilbert space, then $J(x)=\{x\}$, for all $x \in X$;

(2) for each $x \in X, J(x)$ is nonempty closed convex and bounded subset of $X^{*}$;

(3) $J(\lambda x)=\lambda J(x)$, for all $x \in X$ and for all $\lambda \in \mathbb{R}$;

(4) if $X^{*}$ is strictly convex, $J$ is single valued;

(5) if $X$ is strictly convex, $J$ is one to one; that is, $x \neq y \Rightarrow$ $J(x) \cap J(y)=\phi$;

(6) if $X^{*}$ is uniformly convex, then $J$ is uniformly continuous on each bounded set in $X$; that is, for all $\varepsilon>0$ and $\alpha>0$, there is a $\delta=\delta(\varepsilon, \alpha)>0$ such that

$$
\begin{gathered}
\|x\| \leq \alpha, \quad\|y\| \leq \alpha, \\
\|x-y\|<\delta \Longrightarrow\|J(x)-J(y)\|<\varepsilon
\end{gathered}
$$

(note that if $X^{*}$ is uniformly convex, then it is strictly convex, and hence $J$ is single-valued mapping);

(7) if $X$ is reflexive, then $J$ is a mapping from $X$ onto $X^{*}$; that is,

$$
\bigcup\{J(x): x \in X\}=X^{*},
$$


(8) if $X$ is reflexive strictly convex space with strictly convex conjugate space $X^{*}$, then $J$ and $J^{*}$ are one-to-one, onto, and single-valued mappings and

$$
J^{-1}=J^{*}, \quad J J^{*}=I_{X^{*}}, \quad J^{*} J=I_{X},
$$

where $I_{X}$ is the identity mapping on $X$ and $I_{X^{*}}$ is the identity mapping, on $X^{*}$. $[16,19]$

For more properties of the duality mapping, we refer to

Definition 6 (see [1, Definition 2.8.1]). The Banach space $X$ is said to be uniformly smooth if

$$
\rho_{X}^{\prime}(0)=\lim _{t \rightarrow 0} \frac{\rho_{X}(t)}{t}=0,
$$

where $\rho_{X}$ is the modulus of smoothness of $X$ (note that every Hilbert space is uniformly smooth).

Definition 7 (see $[1,17,18]$ ). Let $q>1$ be a real number. A Banach space $X$ is said to be $q$-uniformly smooth if there exist a constant $c>0$ such that $\rho_{X}(t) \leq c t^{q}$, for all $t>0$.

Clearly, every $q$-uniformly smooth is uniformly smooth (note that every Hilbert space is 2-uniformly smooth).

Lemma 8 (see [1, Theorems 2.8.4, 2.8.5, and 2.8.6]). Let $X$ be a Banach space.

(1) $X$ is uniformly smooth if and only if $X^{*}$ is uniformly convex.

(2) $X$ is uniformly convex if and only if $X^{*}$ is uniformly smooth.

(3) If $X$ is uniformly smooth, then $X$ is reflexive.

Lemma 9 (see $[17,18])$. Let $X$ be a Banach space and $p>1$.

(1) If $X$ is $p$-uniformly convex, then $X^{*}$ is $p^{\prime}$-uniformly smooth where $p^{\prime}=p /(p-1)$.

(2) If $X$ is $p$-uniformly smooth, then $X^{*}$ is $p^{\prime}$-uniformly convex where $p^{\prime}=p /(p-1)$. Let

Now, let $X$ be a Banach space and $X^{*}$ its topological dual.

$$
V: X^{*} \times X \longrightarrow \mathbb{R}, \quad V_{*}: X \times X^{*} \longrightarrow \mathbb{R}
$$

be two functions defined by

$$
\begin{gathered}
V(\varphi, x)=\|\varphi\|^{2}-2\langle\varphi, x\rangle+\|x\|^{2}, \\
V_{*}(x, \varphi)=V(\varphi, x) .
\end{gathered}
$$

Based on the functional $V$, the generalized projection of a point $\varphi$ in $X^{*}$ onto a nonempty subset $E$ of $X$ can be defined as follows.
Definition 10 (see [16]). Let $X$ be a Banach space, $E$ a nonempty subset of $X$, and $\varphi \in X^{*}$. If there exists a point $z \in E$ satisfying

$$
V(\varphi, z)=d_{E}^{V}(\varphi)
$$

then $z$ is called a generalized projection of $\varphi$ onto $E$; where $d_{E}^{V}(\varphi)=\inf _{x \in E} V(\varphi, x)$.

The set of all such points is denoted by $\pi_{E}(\varphi)$; that is,

$$
\pi_{E}(\varphi)=\left\{z \in E: V(\varphi, z)=d_{E}^{V}(\varphi)\right\} \subseteq X .
$$

Now, we list in the following theorem some properties of $V$ and $\pi_{E}(\varphi)$ (see $\left.[16]\right)$.

Lemma 11. Let $X$ be a Banach space and $X^{*}$ its topological dual.

(1) $V(J(x), x)=0$.

(2) If $X$ is uniformly convex or uniformly smooth, then

$V(\varphi, x)=0 \Longleftrightarrow \varphi=J(x), \quad \forall x \in X, \varphi \in X^{*}$.

(3) If $X$ is a Hilbert space, then

$$
V(\varphi, x)=\|\varphi-x\|^{2}
$$

(4) If $X$ is reflexive and $E$ is a nonempty closed and convex subset of $X$, then

(a) $\pi_{E}(\varphi) \neq \phi$, for all $\varphi \in X^{*}$;

(b) $X$ is strictly convex if and only if $\pi_{E}(\varphi)$ is singleton, for all $\varphi \in X^{*}$;

(c) if $X$ is also smooth, then, for any given $\varphi \in X^{*}$,

$$
z \in \pi_{E}(\varphi) \Longleftrightarrow\langle-J(z), x-z\rangle \leq 0, \quad \forall x \in E .
$$

Definition 12 (see [4]). Let $X$ be a Banach space with topological dual $X^{*}, E$ a nonempty closed convex subset of $X$, and $z \in X$. The convex normal cone of $E$ at $z$ is defined by

$$
N_{E}(z)=\left\{\varphi \in X^{*}:\langle\varphi, x-z\rangle \leq 0, \forall x \in E\right\} .
$$

For more details about the convex normal cone see $[4,20]$.

Lemma 13 (see [4]). Let $E$ be a nonempty, closed, and convex subset of Banach space $X$, and $z \in X$. Then one has the following.

(1) $N_{E}(z) \cap B_{*}=\partial d_{E}(z)$, where $\partial d_{E}(z)$ is the subdifferential of the function $z \rightarrow d_{E}(z)\left(d_{E}(z)\right.$ is the distance from $z$ to $E$ ).

(2) If $X$ is reflexive and smooth, then

$$
\begin{array}{r}
z \in \pi_{E}(\varphi) \Longleftrightarrow \varphi-J(z) \in N_{E}(z), \\
\forall \varphi \in X^{*}, \forall z \in X .
\end{array}
$$

Also, we need the following four Lemmas. 
Lemma 14 (see [14, Proposition 2.2]). Let $\Omega$ be an open subset in a normal vector space $Z, X$ a Banach space, and $C$ a continuous set-valued mapping defined on $\Omega$ and with nonempty compact convex values in $X$. Let $\left(\varphi_{n}\right)$ be a sequence of $X^{*}$ that converges weakly to $\varphi$ in $X^{*}$ and $\left(x_{n}\right)$ a sequence in $X$ that converges strongly to $x$ in $X$ such that $\left(t_{n}\right)$ a sequence in $\Omega$ that converges to $t$ in I. If $\varphi_{n} \in \partial d_{C\left(t_{n}\right)}\left(x_{n}\right)$, then $\varphi \in \partial d_{C(t)}(x)$.

Lemma 15 (see [14, Lemma 4.1 and Proposition 4.2]). Let $p, q>1, X$ be a $p$-uniformly convex and $q$-uniformly smooth Banach space, and let $E$ be a nonempty bounded subset of $X$; then there exist two constants $\alpha>0, \beta>0$ such that

$$
\alpha\|x-y\|^{p} \leq V(J(x), y) \leq \beta\|x-y\|^{q}, \quad \forall x, y \in E .
$$

Moreover, if $E$, in addition, is closed and $\varphi \in X^{*}$, then

$$
d_{E}^{V}(\varphi)=0 \Longleftrightarrow J^{*}(\varphi) \in E .
$$

Lemma 16 (see [21, Theorem 2.2]). Let $\Omega$ be a subset of $a$ Banach space $X$. Assume that $F:[a, b] \times \Omega \rightarrow 2^{x}$ is a multifunction such that

(1) for every $x \in \Omega$, the multifunction $F(\cdot, x)$ is measurable on $[a, b]$;

(2) for every $t \in[a, b]$, the multifunction $F(t, \cdot)$ is continuous on $\Omega$.

Then, for any measurable function $x(\cdot):[a, b] \rightarrow \Omega$, the multifunction $F(\cdot, x(\cdot))$ is measurable on $[a, b]$.

Lemma 17 (see [21, Lemma 2.3]). Let $X$ be a separable Banach space, $G:[a, b] \rightarrow 2^{X}$ a measurable multifunction, and $z:[a, b] \rightarrow X$ a measurable function. Then for any positive measurable function $r:[a, b] \rightarrow \mathbb{R}^{+}$, there exists a measurable selection $g$ of $G$ such that for almost $t \in[a, b]$

$$
\|g(t)-z(t)\| \leq d(z(t), G(t))+r(t)
$$

\section{Existence of Solutions for Problem (2)}

Theorem 18. Let $p, q>1, X$ a separable $p$-uniformly convex and q-uniformly smooth Banach space, $I=[0, T], T>0, C$ a multifunction defined from $I \times X$ to the family of nonempty closed convex subsets of $X$, and $F$ a multifunction defined from $I \times C_{r} \times C_{r}$ with nonempty closed values in $X^{*}$. Assume that the following hypotheses hold:

$\left(C_{1}\right)$ there is an absolutely continuous function $v: I \rightarrow \mathbb{R}$ and two positive real numbers $\lambda$ and $\gamma$ such that, for all $\xi_{1}, \xi_{2} \in X^{*}$, all $x, y \in X$, and all $t, s \in I$,

$$
\begin{aligned}
& \left|\left(d_{C(t, x)}^{V}\left(\xi_{1}\right)\right)^{1 / q^{\prime}}-\left(d_{C(s, y)}^{V}\left(\xi_{2}\right)\right)^{1 / q^{\prime}}\right| \\
& \quad \leq|v(t)-v(s)|+\lambda\|x-y\|+\gamma\left\|\xi_{1}-\xi_{2}\right\|,
\end{aligned}
$$

where $q^{\prime}=q /(1-q)$;
$\left(C_{2}\right)$ there is a compact subset $K$ of $X^{*}$ such that

$$
J(C(t, x)) \subset K, \quad \forall(t, x) \in I \times X
$$

$\left(F_{1}\right)$ for each $\psi, \varphi \in C_{r}, t \rightarrow F(t, \psi, \varphi)$ is a measurable;

$\left(F_{2}\right)$ there is a function $m \in L^{1}\left(I, \mathbb{R}^{*}\right)$ such that, for all $t \in I$, and for all $\psi_{1}, \varphi_{1}, \psi_{2}, \varphi_{2} \in C_{r}$,

$$
\begin{aligned}
& H\left(F\left(t, \psi_{1}, \varphi_{1}\right), F\left(t, \psi_{2}, \varphi_{2}\right)\right) \\
& \quad \leq m(t)\left(\left\|\psi_{1}-\psi_{2}\right\|+\left\|\varphi_{1}-\varphi_{2}\right\|\right)
\end{aligned}
$$

$\left(F_{3}\right)$ there is a function $p \in L^{1}\left(I, \mathbb{R}^{+}\right)$such that, for all $\psi, \varphi \in$ $C_{r}=C([-r, 0], X)$,

$$
\|F(t, \psi, \varphi)\| \leq p(t)(\|\psi(0)\|+\|\varphi(0)\|), \quad \text { a.e. } t \in I .
$$

Then, for any $\varphi, \psi \in C_{r}$ with $\varphi(0) \in C(0, \psi(0))$, there exist two continuous functions $u:[-r, T] \rightarrow X^{*}$ and $w:$ $[-r, T] \rightarrow X$ such that (2) is satisfied.

Proof. At first, we note that since the Banach space $X$ is $p$-uniformly convex, then it is uniformly convex, and hence by Lemma 2 it is reflexive and strictly convex. Moreover, because $X$ is $q$-uniformly smooth, then, by Lemma 9 , its conjugate $X^{*}$ is $q^{\prime}$-uniformly convex, $q^{\prime}=q /(q-1)$, and hence $X^{*}$ is strictly convex. Then, by Lemmas 5 and 8 , the duality mapping $J$ is single valued, continuous, one-toone, onto, and $J J^{*}=I_{X^{*}}$ and $J^{*} J=I_{X}$. For notational convenience, we take $T=1$. Let $k>0$ such that $K \subset k B^{*}$. Let $n$ be a fixed positive integer and $z: I \rightarrow X^{*}$ a measurable function. In order to make it easier for the reader, we will divide the proof in the following steps.

Step 1. We will construct two continuous functions $u_{n}$ : $[-r, 1] \rightarrow X^{*}$ and $w_{n}:[-r, 1] \rightarrow X$, two functions $\theta_{n}$, $\delta_{n}: I \rightarrow I$ and $f_{n} \in L^{1}\left(I, X^{*}\right)$ such that $u_{n}$ and $w_{n}$ are absolutely continuous function on $I=[0,1]$ and that

(i) $u_{n}(t)=J(\varphi(t)), \quad w_{n}(t)=\psi(t)$, for $t \in[-r, 0]$;

(ii) $f_{n}(t) \in F\left(t, \tau\left(\delta_{n}(t)\right) w_{n}, \tau\left(\delta_{n}(t)\right) J^{*}\left(u_{n}\right)\right)$, for $t \in I$;

(iii) $J^{*}\left(u_{n}\left(\theta_{n}(t)\right)\right) \in C\left(\theta_{n}(t), w_{n}\left(\theta_{n}(t)\right)\right)$ $\cap B^{*}(0, k), \quad$ for $t \in I$;

(iv) $w_{n}(t)=w_{n}\left(\delta_{n}(t)\right)$

$$
+\left(t-\delta_{n}(t)\right) J^{*}\left(u_{n}\left(\delta_{n}(t)\right)\right) \quad \text { for } t \in I,
$$

$w_{n}^{\prime}(t)=J^{*}\left(u_{n}(t)\right), \quad$ a.e. for $t \in I$ 
(v) $\left\|f_{n}(t)-z(t)\right\|$

$$
\begin{aligned}
& \leq d\left(z(t), F\left(t, \tau\left(\delta_{n}(t)\right) w_{n}, \tau\left(\delta_{n}(t)\right) J^{*}\left(u_{n}\right)\right)\right) \\
& +\frac{1}{n}, \quad \text { for } t \in I ;
\end{aligned}
$$

(vi) $\left\|u_{n}^{\prime}(t)-f_{n}(t)\right\|$

$$
\leq \frac{1}{\alpha^{1 / q^{\prime}}}\left[\left|v^{\prime}(t)\right|+\gamma(\|\psi(0)\|+k) p(t)\right]
$$

a.e. for $t \in I$;

(vii) $u_{n}^{\prime}(t)-f_{n}(t)$

$$
\epsilon-N_{C\left(\theta_{n}(t), w_{n}\left(\theta_{n}(t)\right)\right.}\left(J^{*}\left(u_{n}\left(\theta_{n}(t)\right)\right)\right),
$$

a.e. for $t \in I$.

In view of Lemma 15 , there is $\alpha>0$ such that, for all $z_{1}, z_{2} \in$ $\eta B^{*}$, we have

$$
\alpha\left\|z_{1}-z_{2}\right\|^{q^{\prime}} \leq V_{*}\left(J^{*}\left(z_{1}\right), z_{2}\right),
$$

where $\eta=k+(k+\|\psi(0)\|) \int_{0}^{T} p(s) d s$.

For $t \in[-r, 0]$, we define $u_{n}(t)=J(\varphi(t))$ and $w_{n}(t)=$ $\psi(t)$. In order to define $u_{n}$ and $w_{n}$ on $I$, we define a function $e: I \rightarrow \mathbb{R}^{+}$by

$$
e(t):=\frac{1}{\alpha^{1 / q^{\prime}}}\left[\int_{0}^{t}\left(\left|v^{\prime}(s)\right|+\gamma(\|\psi(0)\|+k) p(s)\right) d s+\lambda k\right],
$$

Let us consider a partition of $I=[0,1]$ by the points $t_{i}^{n}=i / n$, $0 \leq i \leq n$. Let $\left.\left.I_{0}^{n}=\left\{t_{0}^{n}\right\}=\{0\}, I_{i+1}^{n}=\right] t_{i}^{n}, t_{i+1}^{n}\right]$, and $0 \leq$ $i \leq n-1$. In view of Lemma 16, there exists a function $f_{0}^{n} \in$ $L^{1}\left(\left[0, t_{1}^{n}\right], X^{*}\right)$ such that $f_{0}^{n}(t) \in F(t, \tau(0) \psi, \tau(0) \varphi)$ and

$$
\begin{array}{r}
\left\|f_{0}^{n}(t)-z(t)\right\| \leq d(z(t), \\
F(t, \tau(0) \psi, \tau(0) \varphi)+\frac{1}{n}, \\
\forall t \in\left[0, t_{1}^{n}\right] .
\end{array}
$$

We put $x_{0}^{n}=\varphi(0) \in C(0, \psi(0))$ and for $t \in I_{1}^{n}$ we define

$$
w_{n}(t):=\psi(0)+\left(t-t_{0}^{n}\right) x_{0}^{n},
$$

$$
\begin{aligned}
u_{n}(t):= & J\left(x_{0}^{n}\right)+\frac{e(t)-e\left(t_{0}^{n}\right)}{e\left(t_{1}^{n}\right)-e\left(t_{0}^{n}\right)} \\
& \times\left(J\left(x_{1}^{n}\right)-J\left(x_{0}^{n}\right)-\int_{t_{0}^{n}}^{t_{1}^{n}} f_{0}^{n}(s) d s\right)+\int_{t_{0}^{n}}^{t} f_{0}^{n}(s) d s,
\end{aligned}
$$

where

$$
x_{1}^{n}:=\pi_{C\left(t_{1}^{n}, w_{n}\left(t_{1}^{n}\right)\right)}\left(J\left(x_{0}^{n}\right)+\int_{t_{0}^{n}}^{t_{1}^{n}} f_{0}^{n}(s) d s\right) .
$$

According to Lemma $11, x_{1}^{n}$ is well defined. By recalling the definition of the generalized projection, we get $x_{1}^{n} \epsilon$ $C\left(t_{1}^{n}, w_{n}\left(t_{1}^{n}\right)\right)$ and

$$
J\left(x_{0}^{n}\right)+\int_{t_{0}^{n}}^{t_{1}^{n}} f_{0}^{n}(s) d s-J\left(x_{1}^{n}\right) \in N_{C\left(t_{1}^{n}, w_{n}\left(t_{1}^{n}\right)\right)}\left(x_{1}^{n}\right) .
$$

As a result of Condition $\left(C_{2}\right),\|\varphi(0)\|=\|J(\varphi(0))\|=$ $\left\|J\left(x_{0}^{n}\right)\right\| \leq k,\left\|J\left(x_{1}^{n}\right)\right\| \leq k$. Moreover, by Condition $\left(F_{3}\right)$

$\left\|\int_{t_{0}^{n}}^{t_{1}^{n}} f_{0}^{n}(s) d s\right\|$

$$
\begin{aligned}
& \leq \int_{t_{0}^{n}}^{t_{1}^{n}} p(s)(\|(\tau(0) \psi)(0)\|+\|(\tau(0) \varphi)(0)\|) d s \\
& \leq\left(\|\psi(0)\|+\| \varphi(0 \|) \int_{t_{0}^{n}}^{t_{1}^{n}} p(s) d s\right. \\
& \leq(\|\psi(0)\|+k) \int_{t_{0}^{n}}^{t_{1}^{n}} p(s) d s .
\end{aligned}
$$

Therefore,

$$
\begin{aligned}
& \left\|J\left(x_{0}^{n}\right)+\int_{t_{0}^{n}}^{t_{1}^{n}} f_{0}^{n}(s) d s\right\| \\
& \quad \leq k+(\|\psi(0)\|+k) \int_{t_{0}^{n}}^{t_{1}^{n}} p(s) d s=\eta .
\end{aligned}
$$

Consequently, by (29) we obtain

$$
\begin{aligned}
\alpha\left\|J\left(x_{1}^{n}\right)-\left(J\left(x_{0}^{n}\right)+\int_{t_{0}^{n}}^{t_{1}^{n}} f_{0}^{n}(s) d s\right)\right\|^{q^{\prime}} \\
\leq V_{*}\left(J^{*}\left(J\left(x_{1}^{n}\right)\right), J\left(x_{0}^{n}\right)+\int_{t_{0}^{n}}^{t_{1}^{n}} f_{0}^{n}(s) d s\right) \\
=\left\|x_{1}^{n}\right\|^{2}-2\left\langle x_{1}^{n}, J\left(x_{0}^{n}\right)+\int_{t_{0}^{n}}^{t_{1}^{n}} f_{0}^{n}(s) d s\right\rangle \\
+\left\|J\left(x_{0}^{n}\right)+\int_{t_{0}^{n}}^{t_{1}^{n}} f_{0}^{n}(s) d s\right\|^{2} \\
=V\left(J\left(x_{0}^{n}\right)+\int_{t_{0}^{n}}^{t_{1}^{n}} f_{0}^{n}(s) d s, x_{1}^{n}\right) \\
=d_{C\left(t_{1}^{n}, w_{n}\left(t_{1}^{n}\right)\right)}^{V}\left(J\left(x_{0}^{n}\right)+\int_{t_{0}^{n}}^{t_{1}^{n}} f_{0}^{n}(s) d s\right) .
\end{aligned}
$$


We note that, from Lemma 15 and the choice $x_{0}^{n}, d_{C\left(t_{0}^{n}, w_{n}\left(t_{0}^{n}\right)\right)}^{V}\left(J\left(x_{0}^{n}\right)\right)=0$. Then, by $\left(C_{1}\right)$ and (36), the last inequality gives us

$$
\begin{aligned}
\alpha^{1 / q^{\prime}} \| & \left(x_{1}^{n}\right)-\left(J\left(x_{0}^{n}\right)+\int_{t_{0}^{n}}^{t_{1}^{n}} f_{0}^{n}(s) d s\right) \| \\
\leq & {\left[d_{C\left(t_{1}^{n}, w_{n}\left(t_{1}^{n}\right)\right)}^{V}\left(J\left(x_{0}^{n}\right)+\int_{t_{0}^{n}}^{t_{1}^{n}} f_{0}^{n}(s) d s\right)\right]^{1 / q^{\prime}} } \\
= & {\left[d_{C\left(t_{1}^{n}, w_{n}\left(t_{1}^{n}\right)\right)}^{V}\left(J\left(x_{0}^{n}\right)+\int_{t_{0}^{n}}^{t_{1}^{n}} f_{0}^{n}(s) d s\right)\right]^{1 / q^{\prime}} } \\
& -\left[d_{C\left(t_{0}^{n}, w_{n}\left(t_{0}^{n}\right)\right)}^{V}\left(J\left(x_{0}^{n}\right)\right)\right]^{1 / q^{\prime}} \\
\leq & \left|v\left(t_{1}^{n}\right)-v\left(t_{0}^{n}\right)\right|+\gamma\left\|\int_{t_{0}^{n}}^{t_{1}^{n}} f_{0}^{n}(s) d s\right\| \\
& +\lambda\left\|w_{n}\left(t_{1}^{n}\right)-w_{n}\left(t_{0}^{n}\right)\right\| \\
\leq & \int_{t_{0}^{n}}^{t_{1}^{n}} v^{\prime}(s) d s+\gamma(\|\psi(0)\|+k) \int_{t_{0}^{n}}^{t_{1}^{n}} p(s) d s+\lambda k .
\end{aligned}
$$

This inequality with (30) leads to

$$
\begin{aligned}
& \left\|J\left(x_{1}^{n}\right)-\left(J\left(x_{0}^{n}\right)+\int_{t_{0}^{n}}^{t_{1}^{n}} f_{0}^{n}(s) d s\right)\right\| \\
& \quad \leq \frac{1}{\alpha^{1 / q^{\prime}}}\left[\int_{t_{0}^{n}}^{t_{1}^{n}}\left(\left|v^{\prime}(s)\right|+\gamma(\|\psi(0)\|+k) p(s)\right) d s+\lambda k\right] \\
& \quad=\left|e\left(t_{1}^{n}\right)-e\left(t_{0}^{n}\right)\right| .
\end{aligned}
$$

Observe that from (33), for $t \in] t_{0}^{n}, t_{1}^{n}[$, we get

$$
\begin{aligned}
u_{n}^{\prime}(t)= & \frac{e^{\prime}(t)}{e\left(t_{1}^{n}\right)-e\left(t_{0}^{n}\right)} \\
& \times\left(J\left(x_{1}^{n}\right)-\left(J\left(x_{0}^{n}\right)+\int_{t_{0}^{n}}^{t_{1}^{n}} f_{0}^{n}(s) d s\right)\right)+f_{0}^{n}(t) .
\end{aligned}
$$

From this equality with (40), we get

$$
\begin{aligned}
& \left\|u_{n}^{\prime}(t)-f_{0}^{n}(t)\right\| \\
& \quad \leq\left|e^{\prime}(t)\right| \\
& \left.\quad=\frac{1}{\alpha^{1 / q^{\prime}}}\left[\left|v^{\prime}(t)\right|+\gamma(\|\psi(0)\|+k) p(t)\right], \quad \forall t \in\right] t_{0}^{n}, t_{1}^{n}[.
\end{aligned}
$$

$$
\text { Note that, from (33), we have }
$$

$$
J\left(x_{0}^{n}\right)+\int_{t_{0}^{n}}^{t_{1}^{n}} f_{0}^{n}(s) d s-J\left(x_{1}^{n}\right) \in N_{C\left(t_{1}^{n}, w_{n}\left(t_{1}^{n}\right)\right)}\left(x_{1}^{n}\right)
$$

Therefore, by (41),

$$
u_{n}^{\prime}(t)-f_{0}^{n}(t) \in-N_{C\left(t_{1}^{n}, w_{n}\left(t_{1}^{n}\right)\right)}\left(J^{*}\left(u_{n}\left(t_{1}^{n}\right)\right)\right), \quad \text { for a.e. } t \in I_{1}^{n}
$$

Moreover, by (30), (33), (36), and (40), for $t, s \in$ $\left[0, t_{1}^{n}\right](s<t)$ we have

$$
\begin{aligned}
& \| u_{n}(t)-u_{n}(s) \| \\
& \leq \frac{e(t)-e(s)}{e\left(t_{1}^{n}\right)-e\left(t_{0}^{n}\right)}\left\|J\left(x_{1}^{n}\right)-J\left(x_{0}^{n}\right)-\int_{t_{0}^{n}}^{t_{1}^{n}} f_{0}^{n}(s) d s\right\| \\
&+\left\|\int_{s}^{t} f_{0}^{n}(\beta) d \beta\right\| \\
& \leq|e(t)-e(s)|+(\|\psi(0)\|+k) \int_{s}^{t} p(\beta) d \beta \\
&= \frac{1}{\alpha^{1 / q^{\prime}}}\left[\int_{s}^{t}\left(\left|v^{\prime}(\beta)\right|+\gamma(\|\psi(0)\|+k) p(\beta)\right) d \beta+\lambda k\right] \\
&+(\|\psi(0)\|+k) \int_{s}^{t} p(\beta) d \beta \\
&= \frac{1}{\alpha^{1 / q^{\prime}}}\left[\int _ { s } ^ { t } \left(\left|v^{\prime}(\beta)\right|+\gamma(\|\psi(0)\|+k) p(\beta)\right.\right. \\
&= \frac{1}{\alpha^{1 / q^{\prime}}}\left[\int _ { s } ^ { t } \left(\left|v^{\prime}(\beta)\right|+(\|\psi(0)\|+k)\right.\right. \\
&\left.\left.+\alpha^{1 / q^{\prime}}(\|\psi(0)\|+k) p(\beta)\right) d \beta+\lambda k\right]
\end{aligned}
$$

this shows that $u_{n}$ is absolutely continuous on $\left[0, t_{1}^{n}\right]$.

We reiterate this process for constructing two sequences $\left(f_{i}^{n}\right),\left(x_{i}^{n}\right), i=0,1, \ldots, n-1$, and two functions $u_{n}$ : $[-r, 1] \rightarrow X^{*}, w_{n}:[-r, 1] \rightarrow X$ such that, for all 
$i=0,1, \ldots, n-1$, the following properties hold:

$$
\begin{aligned}
& f_{i}^{n}(t) \in F\left(t, \tau\left(t_{i}^{n}\right) w_{n}, \tau\left(t_{i}^{n}\right) J^{*}\left(u_{n}\right)\right), \\
& t \in I_{i+1}^{n}, \\
& \left\|f_{i}^{n}(t)-z(t)\right\| \\
& \leq d\left(z(t), F\left(t, \tau\left(t_{i}^{n}\right) w_{n}, \tau\left(t_{i}^{n}\right) J^{*}\left(u_{n}\right)\right)\right)+\frac{1}{n}, \\
& t \in I_{i+1}^{n}, \\
& w_{n}(t)=w_{n}\left(t_{i}^{n}\right)+\left(t-t_{i}^{n}\right) J^{*}\left(u_{n}\left(t_{i}^{n}\right)\right) \text {, } \\
& t \in I_{i+1}^{n} \\
& x_{i+1}^{n}=\pi_{C\left(t_{i+1}^{n}, w_{n}\left(t_{i+1}^{n}\right)\right)}\left(J\left(x_{i}^{n}\right)+\int_{t_{i}^{n}}^{t_{i+1}^{n}} f_{i}^{n}(s) d s\right), \\
& t \in I_{i+1}^{n} \\
& u_{n}(t)=J\left(x_{i}^{n}\right)+\frac{e(t)-e\left(t_{i}^{n}\right)}{e\left(t_{i+1}^{n}\right)-e\left(t_{i}^{n}\right)} \\
& \times\left(J\left(x_{i+1}^{n}\right)-J\left(x_{i}^{n}\right)-\int_{t_{i}^{n}}^{t_{i+1}^{n}} f_{i}^{n}(s) d s\right) \\
& +\int_{t_{i}^{n}}^{t} f_{i}^{n}(s) d s, \quad t \in I_{i+1}^{n}, \\
& \left\|J\left(x_{i+1}^{n}\right)-\left(J\left(x_{i}^{n}\right)+\int_{t_{i}^{n}}^{t_{i+1}^{n}} f_{i}^{n}(s) d s\right)\right\| \leq\left|e\left(t_{i+1}^{n}\right)-e\left(t_{i}^{n}\right)\right|, \\
& \left\|u_{n}^{\prime}(t)-f_{i}^{n}(t)\right\| \\
& \leq\left|e^{\prime}(t)\right|=\frac{1}{\alpha^{1 / q^{\prime}}}\left[\left|v^{\prime}(t)\right|+\gamma(\|\psi(0)\|+k) p(t)\right], \\
& \text { for a.e. } t \in I_{i+1}^{n} \text {, } \\
& u_{n}^{\prime}(t)-f_{i}^{n}(t) \in-N_{C\left(t_{i+1}^{n}, w_{n}\left(t_{i+1}^{n}\right)\right.}\left(J^{*}\left(u_{n}\left(t_{i}^{n}\right)\right)\right), \\
& \text { for a.e. } t \in I_{i+1}^{n} \text {, } \\
& x_{i+1}^{n} \in C\left(t_{i+1}^{n}, w_{n}\left(t_{i+1}^{n}\right)\right) \cap B^{*}(0, k),
\end{aligned}
$$

where $J^{*}\left(u_{n}\right)(t)=J^{*}\left(u_{n}(t)\right)$.

Note that, by (48), for every $i=0,1,2, \ldots, n-1$,

$$
\begin{aligned}
\left\|w_{n}\left(t_{i+1}^{n}\right)\right\| & \leq\left\|w_{n}\left(t_{i}^{n}\right)\right\|+\left|t_{i+1}^{n}-t_{i}^{n}\right|\left\|J^{*}\left(u_{n}\left(t_{i}^{n}\right)\right)\right\| \\
& \leq\left\|w_{n}\left(t_{i}^{n}\right)\right\|+\left|t_{i+1}^{n}-t_{i}^{n}\right| k
\end{aligned}
$$

Then, from this recurrence relation, we can get $\left\|w_{n}\left(t_{i+1}^{n}\right)\right\| \leq\|\psi(0)\|+k, \quad$ for every $i=0,1,2, \ldots, n-1$.
This relation with (46), (54), and Condition $\left(F_{3}\right)$ gives us, for $t \in I_{i+1}^{n}$,

$$
\begin{aligned}
\left\|f_{i}^{n}(t)\right\| & \leq p(t)\left(\left\|\tau\left(t_{i}^{n}\right) w_{n}(0)\right\|+\left\|\tau\left(t_{i}^{n}\right) J^{*}\left(u_{n}\right)(0)\right\|\right) \\
& \leq p(t)\left(\left\|w_{n}\left(t_{i}^{n}\right)\right\|+\left\|J^{*}\left(u_{n}\left(t_{i}^{n}\right)\right)\right\|\right) \\
& \leq p(t)(\|\psi(0)\|+k+k) \\
& =p(t)(\|\psi(0)\|+2 k) .
\end{aligned}
$$

Therefore, by (34), (50), (51), and (57), for $t, s \in I_{i+1}^{n}(s<$ $t$ ) we have

$$
\begin{aligned}
\| u_{n}(t) & -u_{n}(s) \| \\
\leq & \frac{e(t)-e(s)}{e\left(t_{i+1}^{n}\right)-e\left(t_{i}^{n}\right)}\left\|J\left(x_{i+1}^{n}\right)-J\left(x_{i}^{n}\right)-\int_{t_{i}^{n}}^{t_{i+1}^{n}} f_{i}^{n}(s) d s\right\| \\
& +\left\|\int_{s}^{t} f_{i}^{n}(\beta) d \beta\right\| \\
\leq & |e(t)-e(s)|+(\|\psi(0)\|+2 k) \int_{s}^{t} p(\beta) d \beta \\
= & \frac{1}{\alpha^{1 / q^{\prime}}}\left[\int_{s}^{t}\left|v^{\prime}(\beta)\right| d \beta+\gamma(\|\psi(0)\|+k)\right. \\
& +(\|\psi(0)\|+2 k) \int_{s}^{t} p(\beta) d \beta \\
& +(\|\psi(0)\|+2 k) \int_{s}^{t} p(\beta) d \beta . \\
= & \frac{1}{\alpha^{1 / q^{\prime}}}\left[\int_{s}^{t}\left(\left|v^{\prime}(\beta)\right|+\gamma(\|\psi(0)\|+k) p(\beta)\right) d \beta+\lambda k\right] \\
& (\beta k]
\end{aligned}
$$

By addition, the last inequality holds for $t, s \in I$, with $s<$ $t$; hence, $u_{n}$ is absolutely continuous on $I$.

Now, for each positive integer $n$ we define the functions $\theta_{n}, \delta_{n}: I \rightarrow I$ and $f_{n} \in L^{1}\left(I, X^{*}\right)$ by setting

$$
\begin{array}{cc}
\theta_{n}(0)=t_{1}^{n}, & \delta_{n}(0)=0, \\
f_{n}(0)=f_{i}^{n}(0), & \theta_{n}(t)=t_{i+1}^{n}, \\
\delta_{n}(t)=t_{i}^{n}, \quad & f_{n}(t)=f_{i}^{n}(t) \\
& \text { for } t \in I_{i+1}^{n} .
\end{array}
$$

Then obviously, the relations (46), (47), (48), (52), (53), and (54) imply (28)(ii) $\rightarrow$ (vii), respectively.

Step 2 . The sequences $\left(u_{n}\right)$ and $\left(w_{n}\right)$ converge uniformly to continuous functions $u:[-r, 1] \rightarrow X^{*}, w:[-r, 1] \rightarrow X$, respectively, with $u(t)=J(\varphi(t))$ and $w(t)=\psi(t)$ for $t \epsilon$ $[-r, 0]$ and $J^{*}(u(t)) \in C(t, w(t))$ for $t \in I$.

Indeed, by (58) for $t \in I$ we have $\lim _{n \rightarrow \infty} \| u_{n}\left(\theta_{n}(t)\right)-$ $u_{n}(t) \|=0$. Moreover, by (28)(iii), for any $n \geq 1$ and any $t \in I$, 
we have $u_{n}\left(\theta_{n}(t)\right) \in K$. Then, for any $t \in I$, the set $\left\{u_{n}(t)\right.$ : $n \geq 1\}$ is relatively compact subset in $X^{*}$. In addition, from (28)(vi), Condition $\left(F_{3}\right)$, and (57) we get for a.e. $t \in I$

$$
\begin{aligned}
\left\|u_{n}^{\prime}(t)\right\| \leq & \frac{1}{\alpha^{1 / q^{\prime}}}\left[\left|v^{\prime}(t)\right|+\gamma(\|\psi(0)\|+k) p(t)\right] \\
& +\left\|f_{n}(t)\right\| \\
\leq & \frac{1}{\alpha^{1 / q^{\prime}}}\left[\left|v^{\prime}(t)\right|+\gamma(\|\psi(0)\|+k) p(t)\right] \\
& +p(t)\left(\left\|w_{n}\left(\delta_{n}(t)\right)\right\|+\left\|J^{*}\left(u_{n}\left(\delta_{n}(t)\right)\right)\right\|\right) \\
\leq & \frac{1}{\alpha^{1 / q^{\prime}}}\left[\left|v^{\prime}(t)\right|+\gamma(\|\psi(0)\|+k) p(t)\right] \\
& +p(t)(\|\psi(0)\|+2 k) .
\end{aligned}
$$

This, with the absolutely continuity of $u_{n}$, assures the equicontinuity of $u_{n}$. Thus, by Arzela-Ascoli's Theorem, we can select a subsequence of $u_{n}$, again denoted by $u_{n}$, which converges uniformly to an absolutely continuous function $u$ on $I$ such that, for any $t \in I$,

$$
u(t)=J\left(x_{0}^{n}\right)+\int_{0}^{t} u^{\prime}(s) d s
$$

Moreover, $\left(u_{n}^{\prime}\right)$ converges weakly to $u^{\prime} \in L^{1}\left(I, X^{*}\right)$. We extend the definition of $u$ in such a way that $u=J(\varphi)$ on $[-r, 0]$. Obviously, $\left(u_{n}\right)$ converges uniformly to $u$ on $[-r, 1]$. Let us define $u_{n}^{*}(t)=J^{*}\left(u_{n}(t)\right), t \in I$. Since $J^{*}$ is continuous on the compact set $K$ and $u_{n}(t) \in K$ for $t \in I$, then the sequence $\left(u_{n}^{*}\right)$ converges uniformly to a function $u^{*}=J^{*}(u)$. In addition to the fact that $w_{n}^{\prime}(t)=J^{*}\left(u_{n}(t)\right)$, a.e. for $t \in I$, we confirm that the sequence $\left(w_{n}\right)$ converges uniformly to a function $w(t)=\psi(0)+\int_{0}^{t} J^{*}(u(s)) d s, t \in I$. Let us show that $J^{*}(u(t)) \in C(t, w(t))$ for $t \in I$. Note that

$$
\begin{aligned}
\lim _{n \rightarrow \infty}\left\|u_{n}\left(\theta_{n}(t)\right)-u(t)\right\| \leq & \lim _{n \rightarrow \infty}\left\|u_{n}\left(\theta_{n}(t)\right)-u_{n}(t)\right\| \\
& +\lim _{n \rightarrow \infty}\left\|u_{n}(t)-u(t)\right\|=0 .
\end{aligned}
$$

Similarly,

$$
\lim _{n \rightarrow \infty}\left\|w_{n}\left(\theta_{n}(t)\right)-w(t)\right\|=0
$$

Moreover, as $J^{*}\left(u_{n}\left(\theta_{n}(t)\right)\right)=J^{*}\left(u_{n}\left(t_{i+1}^{n}\right)\right)=J^{*}\left(J\left(x_{i+1}^{n}\right)\right)=$ $x_{i+1}^{n} \in C\left(\theta_{n}(t), w_{n}\left(\theta_{n}(t)\right)\right)$, it follows that

$$
d_{C\left(\theta_{n}(t), w_{n}\left(\theta_{n}(t)\right)\right)}^{V}\left(u_{n}\left(\theta_{n}(t)\right)\right)=0, \quad t \in I
$$

Then, from (62), (63), (64), Condition $\left(C_{1}\right)$ and one obtains, for $t \in I$,

$$
\begin{aligned}
& \left(d_{C(t, w(t))}^{V}(u(t))\right)^{1 / q^{\prime}} \\
& \leq \mid\left(d_{C(t, w(t))}^{V}(u(t))\right)^{1 / q^{\prime}} \\
& \quad-\left(d_{C\left(\theta_{n}(t), w_{n}\left(\theta_{n}(t)\right)\right)}^{V}\left(u_{n}\left(\theta_{n}(t)\right)\right)\right)^{1 / q^{\prime}} \mid \\
& \leq\left|v(t)-v\left(\theta_{n}(t)\right)\right| \\
& \quad+\gamma\left\|u_{n}\left(\theta_{n}(t)\right)-u(t)\right\|+\lambda\left\|w_{n}\left(\theta_{n}(t)\right)-w(t)\right\| .
\end{aligned}
$$

By taking the limit as $n \rightarrow \infty$, we conclude that $J^{*}(u(t)) \in C(t, w(t))$ for $t \in I$.

Step 3. For any $t \in I$, the sequence $\tau\left(\delta_{n}(t) u_{n}^{*}\right)$ converges to $\tau(t) u^{*}$, and the sequence $\tau\left(\delta_{n}(t) w_{n}\right)$ converges to $\tau(t) w$ in $C_{r}$. Let $t \in I$. We have

$$
\begin{aligned}
& \left\|\tau\left(\delta_{n}(t)\right) u_{n}^{*}-\tau(t) u^{*}\right\| \\
& \leq\left\|\tau\left(\delta_{n}(t)\right) u_{n}^{*}-\tau(t) u_{n}^{*}\right\|+\left\|\tau(t) u_{n}^{*}-\tau(t) u^{*}\right\| \\
& \leq \sup _{-r \leq s \leq 0}\left\|u_{n}^{*}\left(\delta_{n}(t)+s\right)-u_{n}^{*}(t+s)\right\| \\
& +\left\|\tau(t) u_{n}^{*}-\tau(t) u^{*}\right\| \\
& \leq \sup _{\substack{-r \leq s_{1} \leq s_{2} \leq 1 \\
\left|s_{1}-s_{2}\right| \leq 1 / n}}\left\|u_{n}^{*}\left(s_{1}\right)-u_{n}^{*}\left(s_{2}\right)\right\| \\
& +\left\|\tau(t) u_{n}^{*}-\tau(t) u^{*}\right\| \\
& \leq \sup _{\substack{-r \leq s_{1}, s_{2} \leq 0 \\
\left|s_{1}-s_{2}\right| \leq 1 / n}}\left\|u_{n}^{*}\left(s_{1}\right)-u_{n}^{*}\left(s_{2}\right)\right\| \\
& +\sup _{\substack{-r \leq s_{1} \leq 0 \leq s_{2} \leq 1 \\
\left|s_{1}-s_{2}\right| \leq 1 / n}}\left\|u_{n}^{*}\left(s_{1}\right)-u_{n}^{*}\left(s_{2}\right)\right\| \\
& +\sup _{0 \leq s_{1} \leq s_{2} \leq 1}\left\|u_{n}^{*}\left(s_{1}\right)-u_{n}^{*}\left(s_{2}\right)\right\| \\
& +\left\|\tau(t) u_{n}^{*}-\tau(t) u^{*}\right\| \\
& \leq \sup _{\substack{-r \leq s_{1}, s_{2} \leq 0 \\
\left|s_{1}-s_{2}\right| \leq 1 / n}}\left\|\varphi\left(s_{1}\right)-\varphi\left(s_{2}\right)\right\| \\
& +\sup _{\substack{-r \leq s_{1} \leq 0 \\
\left|s_{1}\right| \leq 1 / n}}\left\|u_{n}^{*}\left(s_{1}\right)-u_{n}^{*}(0)\right\| \\
& +\sup _{\substack{0 \leq s_{2} \leq 1 \\
\left|s_{2}\right| \leq 1 / n}}\left\|u_{n}^{*}(0)-u_{n}^{*}\left(s_{2}\right)\right\| \\
& +\sup _{\substack{0 \leq s_{1} \leq s_{2} \leq 1 \\
\left|s_{1}-s_{2}\right| \leq 1 / n}}\left\|u_{n}^{*}\left(s_{1}\right)-u_{n}^{*}\left(s_{2}\right)\right\|
\end{aligned}
$$




$$
\begin{aligned}
& +\left\|\tau(t) u_{n}^{*}-\tau(t) u^{*}\right\| \\
\leq & 2 \sup _{\substack{-r \leq s_{1}, s_{2} \leq 0 \\
\left|s_{1}-s_{2}\right| \leq 1 / n}}\left\|\varphi\left(s_{1}\right)-\varphi\left(s_{2}\right)\right\| \\
& +\underset{\substack{0 \leq s_{1}, s_{2} \leq 1 \\
\left|s_{1}-s_{2}\right| \leq 1 / n}}{2 \operatorname{su}_{n}}\left\|u_{n}^{*}\left(s_{1}\right)-u_{n}^{*}\left(s_{2}\right)\right\| \\
& +\left\|\tau(t) u_{n}^{*}-\tau(t) u^{*}\right\| .
\end{aligned}
$$

By the continuity of $\varphi$, the uniform convergence of $u_{n}^{*}$ towards $u^{*}$, and the preceding estimate, we get

$$
\lim _{n \rightarrow \infty}\left\|\tau\left(\delta_{n}(t)\right) u_{n}^{*}-\tau(t) u^{*}\right\|=0 .
$$

Similarly, we can show that $\lim _{n \rightarrow \infty} \| \tau\left(\delta_{n}(t)\right) w_{n}-$ $\tau(t) w \|=0$.

Step 4 . The sequence $\left(f_{n}\right)$ converges pointwise to a function $f \in L^{1}\left(I, X^{*}\right)$ satisfying $f(t) \in F\left(t, \tau(t) w, \tau(t) u^{*}\right), t \in I$.

Let $t \in I$ be fixed. In view of $(28)(\mathrm{v})$ and Condition $\left(F_{2}\right)$, we obtain for $n \geq 1$

$$
\begin{aligned}
& \left\|f_{n+1}(t)-f_{n}(t)\right\| \\
& \leq H\left(F\left(t, \tau\left(\delta_{n}(t)\right) w_{n}, \tau\left(\delta_{n}(t)\right) u_{n}^{*}\right),\right. \\
& \left.\quad F\left(t, \tau\left(\delta_{n+1}(t)\right) w_{n+1}, \tau\left(\delta_{n+1}(t)\right) u_{n+1}^{*}\right)\right)+\frac{1}{n+1} \\
& \leq m(t)\left(\left\|\tau\left(\delta_{n}(t)\right) w_{n}-\tau\left(\delta_{n+1}(t)\right) w_{n+1}\right\|\right. \\
& \left.\quad+\left\|\tau\left(\delta_{n}(t)\right) u_{n}^{*}-\tau\left(\delta_{n+1}(t)\right) u_{n+1}^{*}\right\|\right) \\
& +\frac{1}{n+1} .
\end{aligned}
$$

From Step 3, the right-hand side of (68) tends to zero when $n \rightarrow \infty$. Hence, $\left(f_{n}(t)\right)$ is a Cauchy sequence in $X^{*}$; then it converges pointwise to a function $f \in L^{1}\left(I, X^{*}\right)$. Moreover, by (28)(v) and Condition $\left(F_{2}\right)$, we get

$$
\begin{aligned}
& d\left(f(t), F\left(t, \tau(t) w, \tau(t) u^{*}\right)\right) \\
& \leq\left\|f(t)-f_{n}(t)\right\| \\
& +H\left(F\left(t, \tau(t) w, \tau(t) u^{*}\right),\right. \\
& \left.\quad F\left(t, \tau\left(\delta_{n}(t)\right) w_{n}, \tau\left(\delta_{n}(t)\right) u_{n}^{*}\right)\right) \\
& \leq\left\|f(t)-f_{n}(t)\right\|+m(t) \\
& \times\left(\left\|\tau(t) w-\tau\left(\delta_{n}(t)\right) w_{n}\right\|\right. \\
& \left.+\left\|\tau(t) u^{*}-\tau\left(\delta_{n}(t)\right) u_{n}^{*}\right\|\right) .
\end{aligned}
$$

Again, by Step 3, the right-hand side of this inequality tends to zero when $n \rightarrow \infty$. Hence, $f(t) \in$ $F\left(t, \tau(t) w, \tau(t) J^{*}(u)\right)$, and $t \in I$.
Step 5. For almost $t \in I, u^{\prime}(t)-f(t) \in-N_{C(t, w(t))}\left(J^{*}(u(t))\right)$.

We note the weak convergence of $u_{n}^{\prime}-f_{n}$ towards $u^{\prime}-f$ in $L^{1}\left(I, X^{*}\right)$, and Mazur's Lemma gives us

$$
u^{\prime}(t)-f(t) \in \bigcap_{n} \overline{\operatorname{Co}}\left\{u_{m}^{\prime}(t)-f_{m}(t): m \geq n\right\},
$$

for a.e. $t \in I$.

Fix any $t$ such that the preceding relation is satisfied, and consider $x \in X$. The last relation above yields

$$
\left\langle u^{\prime}(t)-f(t), x\right\rangle \leq \inf _{n} \operatorname{Sup}_{m \geq n}\left\langle u_{m}^{\prime}(t)-f_{m}(t), x\right\rangle .
$$

Moreover, the relations (28)(vi) and (vii) tell us for a.e. $t \in I$

$$
\begin{aligned}
u_{n}^{\prime}(t) & -f_{n}(t) \\
& \left.\in-N_{C\left(\theta_{n}(t), w_{n}\left(\theta_{n}(t)\right)\right)}\right)^{*}\left(u_{n}\left(\theta_{n}(t)\right)\right) \cap B^{*}\left(0, e^{\prime}(t)\right),
\end{aligned}
$$

where the function $e$ is defined by (34). Hence, by Lemma 13, for a.e. $t \in I$, we have

$$
\begin{aligned}
u_{n}^{\prime}(t) & -f_{n}(t) \\
& \in-e^{\prime}(t) \partial d_{C\left(\theta_{n}(t), w_{n}\left(\theta_{n}(t)\right)\right)}\left(J^{*}\left(u_{n}\left(\theta_{n}(t)\right)\right)\right) .
\end{aligned}
$$

In view of (63) and (64), for $x \in X$ and a.e. $t \in I$, one obtains

$$
\begin{aligned}
\left\langle u^{\prime}(t)\right. & -f(t), x\rangle \\
\leq & \lim _{n \rightarrow \infty} \operatorname{Sup}\left\langle u_{m}^{\prime}(t)-f_{m}(t), x\right\rangle \\
\leq & e^{\prime}(t) \lim _{n \rightarrow \infty} \operatorname{Sup} \sigma x, \\
& \quad-\partial d_{C\left(\theta_{n}(t), w_{n}\left(\theta_{n}(t)\right)\right)}\left(J^{*}\left(u_{n}\left(\theta_{n}(t)\right)\right)\right),
\end{aligned}
$$

where $\sigma$ is the support function. From the uppersemicontinuity property, Lemma 14 and the last relation above yield for $x \in X$ and a.e. $t \in I$

$$
\left\langle u^{\prime}(t)-f(t), x\right\rangle \leq e^{\prime}(t) \sigma\left(x,-\partial d_{C(t, w(t))} J^{*}(u(t))\right) .
$$
get

As the set $\partial d_{C\left(t, w_{n}(t)\right)}\left(J^{*}(u(t))\right)$ is closed and convex, we

$$
u^{\prime}(t)-f(t) \in-N_{C(t, w(t))}\left(J^{*}(u(t))\right), \quad \text { for a.e. } t \in I .
$$

This proves the claim.

Finally, by Steps 4 and 5 we have for almost $t \in I$

$$
\begin{aligned}
u^{\prime}(t) & \in-N_{C(t, w(t))}\left(J^{*}(u(t))\right) \\
& +F\left(t, \tau(t) w, \tau(t) J^{*}(u(t))\right),
\end{aligned}
$$

and the proof is complete. 


\section{Conclusion}

In this paper, existence problem of solution of a functional sweeping process in Banach space with noncompact perturbation has been considered. Some sufficient conditions have been obtained. The importance of this work due to the fact that the values of the perturbation are not necessarily convex or compact and the considerable space is a Banach space.

\section{Acknowledgment}

The authors gratefully acknowledge the Deanship of Scientific Research, King Faisal University of Saudi Arabia, for its financial support concerning this research project.

\section{References}

[1] R. P. Agarwal, D. O'Regan, and D. R. Sahu, Fixed Point Theory for Lipschitzian-Type Mappings with Applications, Springer, London, UK, 2009.

[2] J.-P. Aubin and A. Cellina, Differential Inclusions: Set-Valued Maps and Viability Theory, vol. 264, Springer, Berlin, Germany, 1984.

[3] A. Bressan, "Differential inclusions and the control of forest fires," Journal of Differential Equations, vol. 243, no. 2, pp. 179207, 2007.

[4] F. H. Clarke, Optimization and Nonsmooth Analysis, Canadian Mathematical Society Series of Monographs and Advanced Texts, John Wiley \& Sons, New York, NY, USA, 1983.

[5] J.-J. Moreau, "Evolution problem associated with a moving convex set in a Hilbert space," Journal of Differential Equations, vol. 26, no. 3, pp. 347-374, 1977.

[6] M. Aitalioubrahim, "On noncompact perturbation of nonconvex sweeping process," Commentationes Mathematicae Universitatis Carolinae, vol. 53, no. 1, pp. 65-77, 2012.

[7] C. Castaing, A. G. Ibrahim, and M. Yarou, "Some contributions to nonconvex sweeping process," Journal of Nonlinear and Convex Analysis, vol. 10, no. 1, pp. 1-20, 2009.

[8] M. Bounkhel, "Existence and uniqueness of some variants of nonconvex sweeping processes," Journal of Nonlinear and Convex Analysis, vol. 8, no. 2, pp. 311-323, 2007.

[9] M. Bounkhel and D.-L. Azzam, "Existence results on the second-order nonconvex sweeping processes with perturbations," Set-Valued Analysis, vol. 12, no. 3, pp. 291-318, 2004.

[10] M. Bounkhel and L. Thibault, "Nonconvex sweeping process and prox-regularity in Hilbert space," Journal of Nonlinear and Convex Analysis, vol. 6, no. 2, pp. 359-374, 2005.

[11] J. F. Edmond and L. Thibault, "BV solutions of nonconvex sweeping process differential inclusion with perturbation," Journal of Differential Equations, vol. 226, no. 1, pp. 135-179, 2006.

[12] L. Thibault, "Requalarization of non-convex sweeping process in Hilbert Spaces," Set-Valued Analysis, vol. 16, no. 23, pp. 319333, 2008.

[13] L. Thibault, "Sweeping process with regular and nonregular sets," Journal of Differential Equations, vol. 193, no. 1, pp. 1-26, 2003.

[14] M. Bounkhel and R. Al-Yusof, "First and second order convex sweeping processes in reflexive smooth Banach spaces," SetValued and Variational Analysis, vol. 18, no. 2, pp. 151-182, 2010.
[15] A. G. Ibrahim and F. A. Aladsani, "Second order evolution inclusions governed by sweeping process in Banach spaces," Le Matematiche, vol. 64, no. 2, pp. 17-39, 2009.

[16] Ya. I. Al'ber, "Generalized projection operators in Banach spaces: properties and applications," Functional-Differential Equations, vol. 1, pp. 1-21, 1994.

[17] R. Deville, G. Godefroy, and V. Zizler, Smoothness and Renormings in Banach Spaces, vol. 64 of Pitman Monographs and Surveys in Pure and Applied Mathematics, Longman Scientific \& Technical, Harlow, UK, 1993.

[18] J. Diestel, Geometry of Banach Spaces: Selected Topics, vol. 485 of Lecture Notes in Mathematics, Springer, Berlin, Germany, 1975.

[19] W. Takahashi, Nonlinear Functional Analysis, Yokohama Publishers, Yokohama, Japan, 2000.

[20] C. Castaing and M. Valadier, Convex Analysis and Measurable Multifunctions, vol. 580 of Lecture Notes in Mathematics, Springer, Berlin, Germany, 1977.

[21] Q. J. Zhu, "On the solution set of differential inclusions in Banach space," Journal of Differential Equations, vol. 93, no. 2, pp. 213-237, 1991. 


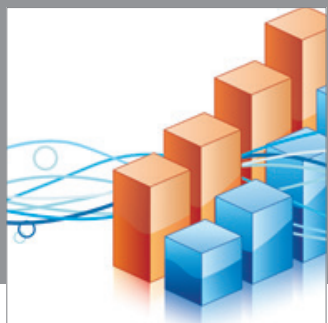

Advances in

Operations Research

mansans

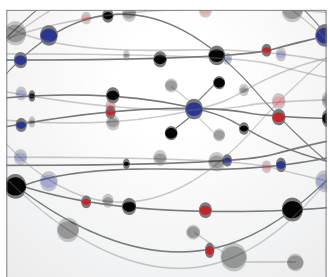

The Scientific World Journal
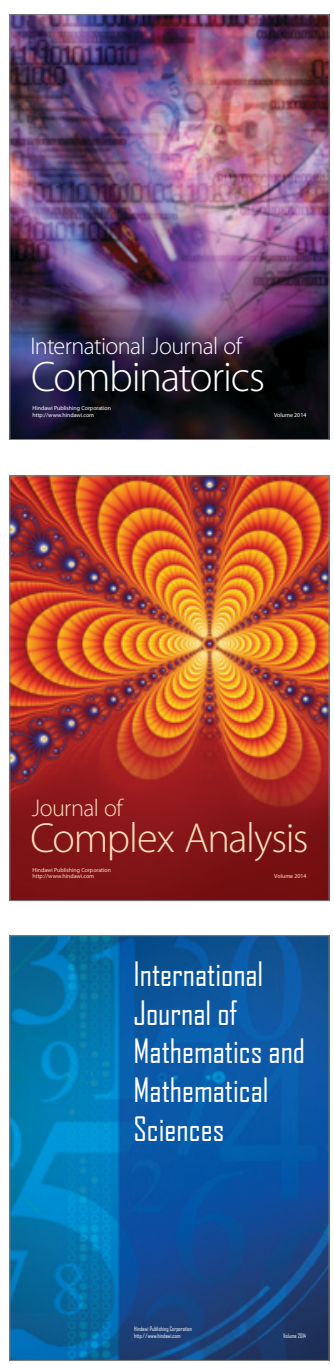
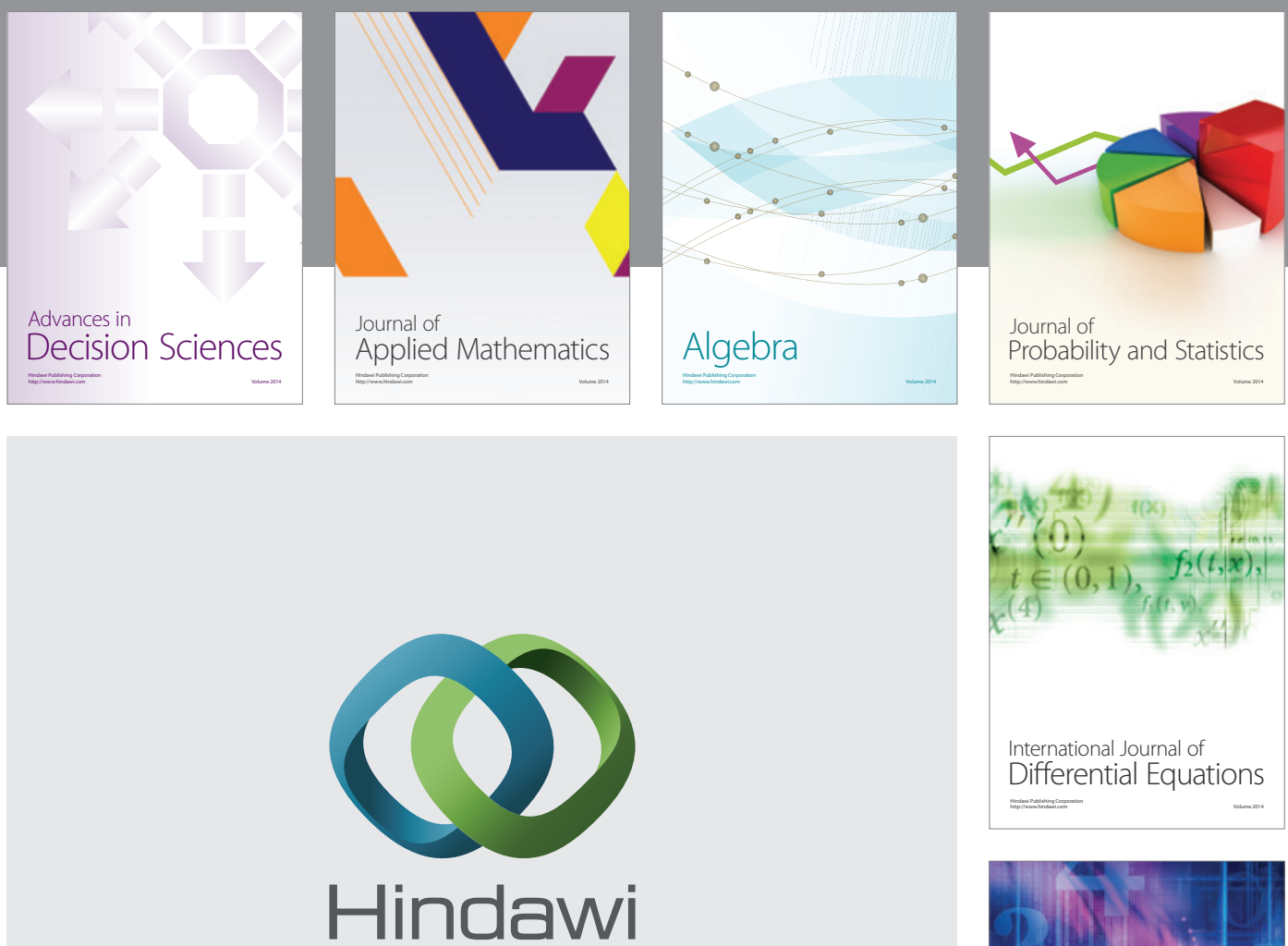

Submit your manuscripts at http://www.hindawi.com
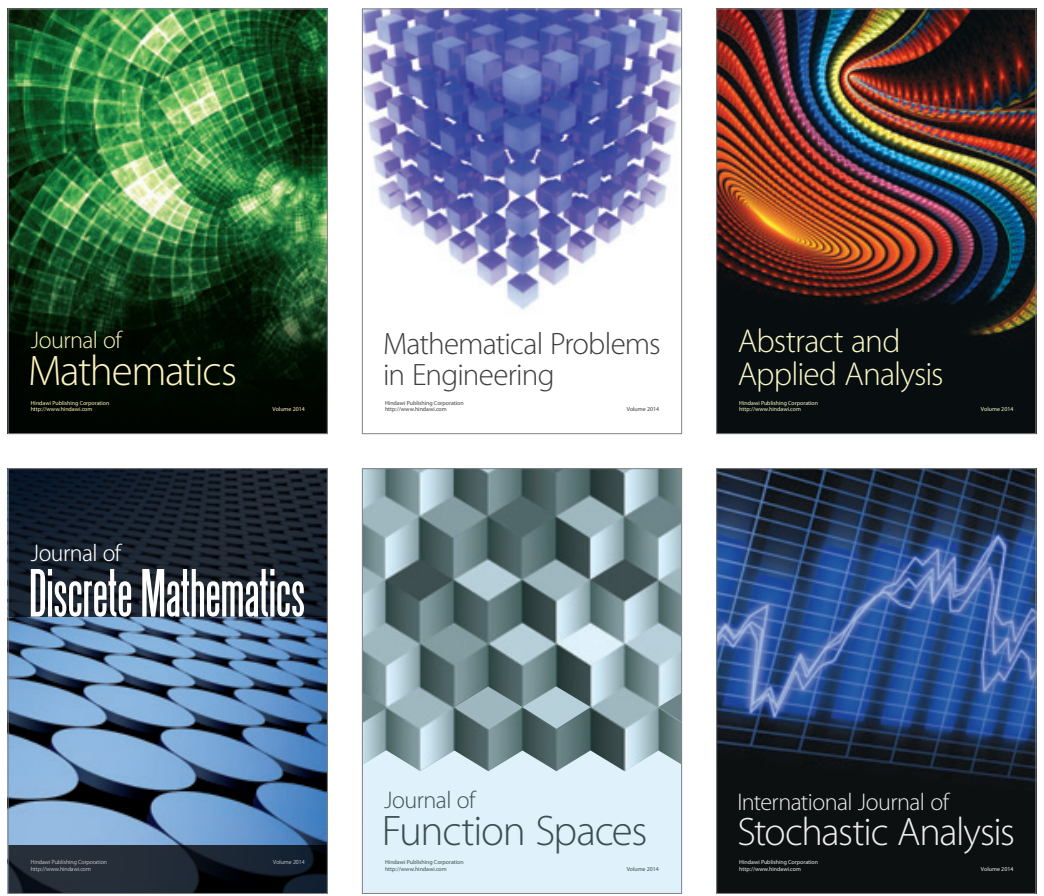

Journal of

Function Spaces

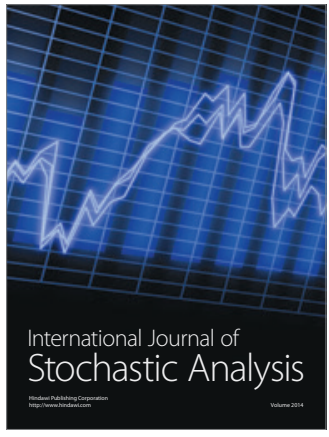

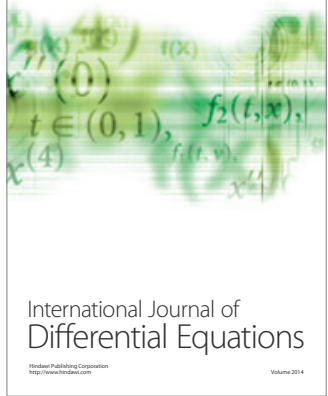
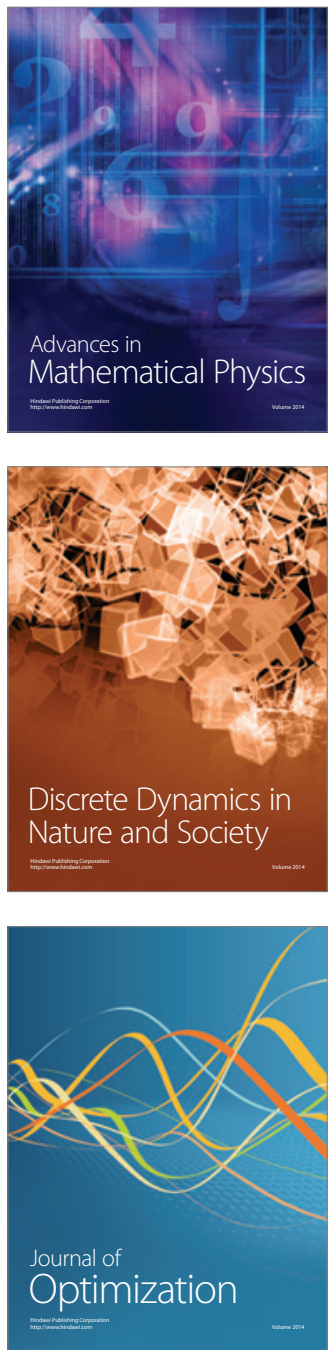